\title{
Contextual Categorization: a Mechanism Linking Perception and Knowledge in Modeling and Simulating Perceived Events as Actions
}

\author{
Elisabetta Zibetti ${ }^{1}$, Vicenç Quera ${ }^{2}$, Francesc Salvador Beltran ${ }^{2}$, Charles Tijus ${ }^{1}$, \\ ${ }^{1}$ Laboratoire CNRS- ESA 7021 de Cognition et Activités Mentales Finalisées, \\ Université de Paris 8. \\ http://www.ipt.univ-paris8.fr/ psycog/ \\ Université de Paris 8, 2, rue de la Liberté, \\ 93526 Saint-Denis Cedex 02 FRANCE \\ Tel: 0033149406479 \\ Fax: 0033149406754 \\ ${ }^{2}$ GTICC (Grup de Tecnologia Informàtica en Ciències del Comportament), \\ Universitat de Barcelona. \\ http://www.ub.es/comporta/gticc.htm \\ ezibetti@univ-paris8.fr \\ vquera@psi.ub.es \\ fsalvador@psi.ub.es \\ tijus@univ-paris8.fr
}

\begin{abstract}
The specific objective of this paper is to introduce the computer model ACACIA (Action by Contextually Automated Categorizing Interactive Agents) capable of simulating the way in which context is taken into account for the interpretation of perceived actions elaborated by a number of autonomous moving agents in a bidimensional space. With this in mind, we will examine some different modeling approaches in Artificial Intelligence and Artificial Life and emphasize the strong and weak points of each approach in relation to the set of issues addressed by our theory based on Contextual Categorization. Second, we provide a theoretical explanation of how contextual categorization accounts for temporal and environmental context to interpret ongoing situations in terms of perceived action. Finally, we describe the computer implementation of ACACIA, and we propose a preliminary simulation of a simple situation using StarLogo software.
\end{abstract}

Keywords: PERCEIVED EVENTS, ACTION, CONTEXT, CATEGORIZATION, MODELLING 


\section{Introduction}

The question we address here is the manner in which context intervenes in the interaction between agents, which is to say, when each agent perceives and interprets the actions of other agents in order to take action on its own. To this end, we will first present the interpretation of perceived action, followed by a discussion of different models developed in the Cognitive Sciences. Lastly, we will present our approach based on Contextual Categorization and Artificial Life which takes the form of the computer model, ACACIA (Action by Contextually Automated Categorizing Interactive Agents) a joint development of the GTICC team (Computer Technology for the Behavioral Science Research Group, University of Barcelona) and the research team at the Laboratoire CNRS- ESA 7021, Cognition et Activités Mentales Finalisées, (University of Paris 8).

\section{The interpretation of perceived action}

What mechanisms and processes do we need in order to model the understanding of seen events in terms of action? The interpretation of perceived action is one of the highest and most complex activities carried out by the human brain. It involves both basic and higher-level functions (e.g., detection of objects and events vs. verbalizing actions which are not directly perceived in the environment but inferred), and it requires inter-relating different kinds of knowledge to the perception of an environment that continues changing.

In terms of bottom-up processing, understanding a situation of daily life such as a person crossing a busy street consists in being able to interpret the spatiotemporal relations between the objects in the world (e.g., a person, a vehicle, a street and its sidewalks) as actions done by agents [28, 29, 30].

In terms of top-down processing, understanding such daily situations requires a number of cognitive components such as the observer's attribution of goals and intentions to the other agents, drawing causal links between the different events, predicting future events, and thus activating knowledge. Finally, this understanding allows the observer to anticipate action and perhaps interact (e.g., to help a visually handicapped person cross the street).

How can the problem of matching the bottom-up perceptual input data and the top-down knowledge data be solved? Because the world is not made up of a set of objects and events with conceptual counterparts in the mind, the notions of "object" and "event" need defining in the context of the interaction between the observer and the changing environment in which its stands, in order to account for the observer's ability to understand and interpret new situations. But, what kind of knowledge can be matched to a changing and somewhat unpredictable world?

Our stand, like Barsalou's "ad hoc categories" [3], is based on the idea that actions are interpretations based on perception: we place ourselves in a perspective which accords a decisive role to the physical properties of objects and to the perceived relations between objects in a context (environmental and temporal) in order to 
interpret events as actions. The mechanism we hypothesize as being responsible for the emergence of action from events is contextual categorization.

The process of building contextual categories creates a circumstantial representation, which can be seen as a short-term database. Contextual categories are then used in order to infer new properties. These properties are goals from which action can be derived and attributed to agents. Consider for instance the perception of someone moving through space. This person will not simply be seen as someone moving but instantiated in the contextual category of "walking persons". In contrast, a moving ball will be instantiated as a "rolling ball". "Walking persons" are people that have the goal "of going somewhere", whereas "rolling balls" "move in the direction they were kicked". If the direction in which the object is moving is toward a parked car, instantiated as being a "car", then a superordinate contextual category will be built to catch the two objects "walking person" and "car" or "rolling ball" and "car". This superordinate category will factorize what is common to subordinate categories which, respectively, are "to drive" or "to be under". Thus, the "walking person" can be attributed the goal of "using the car", whereas the "rolling ball" can be attributed the property "will disappear under the car".

Note that this process of contextual categorization is neither construed as a learning process, nor as a form of organizing and storing knowledge in long term memory, but as an active process that allows making inferences while adapting to the current state of the situation. This extension of the concept of categorization to include a "mechanism linking ongoing perception and knowledge structures" is what we believe will allow accounting economically and ecologically for dynamic spatial and temporal contextual effects.

In our approach based on categorization, context is central. Our definition of context is Turner's [26] definition of "the world state", which is to say, "the state of the agent's world at some particular time: i.e., all features of the world, including all objects in existence, their properties and internal states, and the relationships between them" (p.376). Also central to our approach, is the fact that we do not separate the target from the context as is usually done: the target emerges from the contextual categories. Each objet is processed in relation to the other objects in the context. Thus, all objects are similarly processed and instantiated in circumstantial categories which are, in turn, grouped into superordinate categories. As concerns our topic, spatial and temporal coordinates are determining factors in understanding what is taking place.

Let us now present and discuss some of the existing Artificial Intelligence models which are, to our knowledge, the only kind of model proposed for simulating the interpretation of perceived action. We will then provide a theoretical explanation of how contextual categorization accounts for the role of temporal and environmental context in interpreting ongoing situations in terms of perceived action. Finally we will describe a computer implementation of our model, ACACIA, and propose a preliminary simulation of a simple situation using StarLogo software [18].

\section{Some existing models for interpreting perceived actions}

As far as we know, few models have been proposed for modeling the interpretation of perceived action. A first type of computation model arises at least in two major 
subareas of Artificial Intelligence (AI), (i) natural language processing [e.g.; 22] and (ii) computer vision techniques for object and movement recognition [e.g.; 1, 9, 10] and probabilistic plan recognition $[9,10]$. These models, like other models traditionally developed in AI., adopt a primarily top down perspective for studying cognitive processes. These are closed systems using pre-defined knowledge for building representations of a situation which is often posited without the constraints constituted by a changing spatial (environmental) and temporal context. In contrast, a second type of computational model arises from the Artificial Life (AL) paradigm [e.g.; 11, 5, 12, 16, 17]. This more recent approach takes an evolutionist, situated and bottom-up perspective in which the data furnished by the environment have the priority for studying the adaptive capacities of living beings and robots. In this section, we will briefly present three existing AI models intended for simulating perceived action and their limits as we see them. We then introduce the Artificial Life approach and what it can contribute to the discussion.

\subsection{The Thibadeau model: a faitfhful mapping of natural laguage theory}

One of the first propositions for modeling perceived action was put forward by Thibadeau [22]. His model is derived from Miller and Johnson-Laird's "componential" theory [13] in which complex actions are built up from primitive ones (action primitives) with the addition of traits such as direction, agent, intentionality, manner, etc. This model takes the transformations rather than the nature of actions into account. But Thibadeau does not consider the fact that changing the objects involved in the situation may change the interpretation of the action. If the processing of the objects present in the situation where events take place is secondary to comprehension, then how does one explain that the same series of events in a different context can result in interpretations that differ in terms of action? [28, 30]. In fact, we believe that the physical and functional properties of the objects that are involved in a perceived situation are determining factors for comprehension. The functional properties of an object, inferred from the relational properties established between the objects in the context, are the ones that allow accessing predictions as to the actions the object can carry out or be submitted to as well as the manner in which it can be interacted with $[4,26,25,21,27,6]$.

\subsection{The Ayes \& Shah model based on object and movement recognition}

In order to account for contextual properties, Ayes and Shah [1] developed a system capable of recognizing the actions of different persons acting within a defined environment (e.g., an office). Their system represents an innovation in the field of AI because it uses context for recognizing actions that are difficult to model, such as "picking up the phone", "using a computer", etc. The context in which the events take place is stored in the form of a knowledge base relative to the scene: where the entrances are located, the objects present and the manner in which they are usually used. What we believe is relevant and to be emphasized in this approach is that 
information about the function of objects is fundamental for allowing the system to recognize changes in space and time concerning one or several objects as an action.

Nonetheless, the information carried by the function of an object and its relation to the environmental context is not always obvious or enough for furnishing correct interpretations. The interpretation of events as actions is also heavily dependent on the goals attributed to the actors and to the temporal context.

\subsection{The Intille \& Bobick probabilistic model based on object and movement recognition and probabilistic plan recognition.}

The probabilistic model for the representation and visual recognition of complex action presented by Intille and Bobick [10] and Intille [9] includes a particularly interesting formalization of the effects of temporal context. Action is represented using temporal graphs. "The primitive in these graphs are agent-based belief networks that can recognize agent goal by probabilistic integration of visual evidence" (p.80).

Intille and Bobick simulated representation and recognition of complex actions in American football. They developed algorithms that integrate contextual domain knowledge and computer vision feature detection in order to interpret and describe events in video sequences. Their system automatically labels states and actions such as "passing" and "blocking" using probabilistic models. Their studies are among the most comprehensive in the field of modeling and simulating perceived action. However, like most AI models, theirs requires a pre-defined knowledge base, very well defined as to the variables involved in football. This is why the model can not explain how someone who knows nothing about this sport may begin to understand merely by watching, start attributing goals to the players, extract the rules and eventually anticipate the players' strategies.

\subsection{Limitations of this kind of system from a psychological point of view}

What is indisputably fascinating about the models developed in AI is that they work so well in some well defined domains. Indeed, by applying astute methods for solving problems such as the detection of objects and movements, they are fairly efficient in surveillance tasks in pre-coded environments. However, when the spatial and temporal variables of a context can not be completely mastered and coded beforehand, their performance levels decline drastically due to their lack of psychological relevance.

What, from a psychological point of view, is probably the most "bothersome" limitation of this kind of system, particularly for the proponents of the importance of contextual data (temporal and visual-perceptive), is their heavily topdown approach to what goes into a representation. In the "closed system" paradigm, context tends to be defined as static and predetermined. This is why these systems, unlike human observers, are so difficult to adapt to a world which is only partially predictable. 


\subsection{Bottom-up and top-down approaches to interpreting perceived actions}

A perspective emphasizing bottom-up processing would seem to be more adequate for accounting for context. In this respect, our approach is similar to Artificial Life's [11, $5,12,16,17]$. AL postulates that the mechanisms underlying adaptive behavior must necessarily be studied and conceived in situ, in other words, the goals the agent is trying for and the opportunities the environment affords for doing so must be accounted for. This kind of approach is highly ecological and parsimonious. Though our approach has much in common with AL's (i.e., parsimony, active learning as a function of goal, adaptability, etc...), it is obvious that simulating the "adaptive behavior" of an observer and potential agent would involve making our system capable of acquiring knowledge about objects in the world and successfully linking contextual data to acquired knowledge.

According to our conception, an observer's representation is not internal, stored, complete and activated for purposes of comprehension (I.A.). Neither is it absent nor is it distributed throughout the successive algorithms which allow the agent to show adaptive behavior without prior knowledge of the objects it will encounter $(\mathrm{AL})$. In our approach, representations are put together in an "ad hoc" and dynamic manner in an interface which allows access to stored knowledge through the physical properties of objects and their reciprocal relationships to the other objects in the environment. In this way, the role of knowledge is reduced, it comes into play in processing objects as a source of information, it is not a knowledge base activated all at once by top down processes. Additionally, because comprehension is highly dependent on a constantly changing context, an adaptive agent should be capable of economically assimilating the changes as they occur.

For the problem at hand, it is necessary to postulate the existence of a mechanism linking actual-perception and knowledge structures at each moment of a current situation. The mechanism should account for temporal context by providing comparisons between the past perceptual configurations of the environment $(t-1)$, the current configuration of the world $(\mathrm{t})$, plus inferences about the future activated in the mind of the observer $(t+1)$ which also modify the representation of the situation.

In contrast to fishing in a knowledge base that is previously organized and structured and which contains information about actions, the construction of contextual categories seems to offer the link between actual perception and knowledge that allows activating inferences based on a set of actions relevant to understanding a given situation.

\section{A Contextual Categorization Approach}

If perception is a purposeful activity involving the pick-up of relevant environmental patterns $[7,8]$, then all the components leading to an action are not necessarily actually perceived: they may be inferred from other temporal and spatial contextual components as well as from knowledge about the properties of objects. We interpret this principle of inference by proposing that the construction of a representation of a perceived evolving situation is a sequential process during which objects are categorized on line. As objects appear and disappear and as their properties change, 
contextual categories are created and modified. The categorization, both of objects and of events, generates inferences through the implication of categories and is thus responsible for goal attribution and the emergence of a specific interpretation. For instance, if an object that occupies place A, disappears and appears at place B, then it can be categorized as a moving object, either animate or being pushed or propelled. If animate, then the movement is intentionally directed. If the movement is in the direction of another object, then the object can be instantiated in the category of an animate object approaching another object, for instance "following" or "chasing" [28, 30].

The mechanisms involved in this process are modeled using the Dynamic Allocation of Meaning Model (C.A.D.S., Categorisation et Assignation Dynamique de Signification) $[23,24,29]$. This model proposes an explanation of the manner in which temporal information and the perceived properties of a situation interact by constructing contextual categories. Through an approach based on contextual categorization that is heavily dependant on the spatiotemporal properties of objects, the representation is no longer centralized but distributed across a dynamic interface between the observer and the world context being experienced.

\subsection{On line contextual spatial categorization using a Galois lattice}

On line contextual categorization using a Galois lattice ${ }^{1}$ is a new research topic that we have applied to perceived action [29]. Consider the four frames of a short movie involving three objects (figure 1). The Galois Lattice (figure 2) of frame 1 has four categories, whereas the lattice of frame 2 has six categories. In the four frames, object $a$ does not change (same shape, color and place). However, in the Galois lattice of frame 1, object $a$ belongs to two categories and in the Galois lattice of frame 2, it belong to three categories. Notice that object $a$ is not moving (figure 1) but is nevertheless affected to contextual categories because of relational and contextual properties (figure 2).

\footnotetext{
${ }^{1}$ The Galois lattice [2] is a hierarchy of categories with transitivity, assymetry and irreflexivity, when given the $\mathrm{O}_{n} \times \mathrm{P}_{\mathrm{m}}$ boolean matrice which indicates for each of the $n$ objects $\mathrm{O}$, whether or not it has each of the $\mathrm{m}$ properties $\mathrm{P}$. The maximum number of categories is either $2^{\mathrm{n}-1}$, or $\mathrm{m}$ if $\mathrm{m}<2^{\mathrm{n}-1}$, in a lattice whose complexity depends on the way properties are distributed over objects. The hierarchy of categories has the inheritance principle of taxonomies: for one object, to belong to one category is to belong to the superordinate ones, having the properties of one category means having the properties of the superordinate categories. In order to build the Galois lattice network of object 'properties, objects are on the first colum and properties on the first line of a binary table. When the object posses this properties it is coodied by " 1 " in a boolean table, when it does not posses that properties it is coodied by " 0 ". The Galois lattice are automatically constructs by STONE software [15]
} 


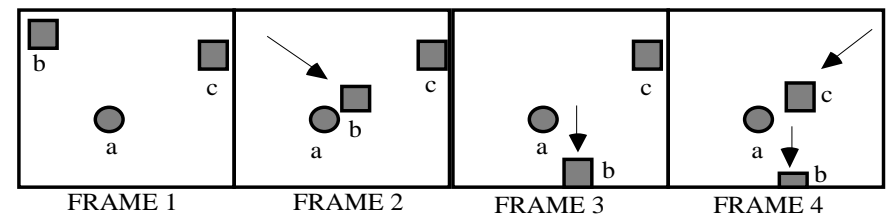

Figure 1. Four frames of a short animated film which shows two rectangles approaching a circle, touching it and then leaving the scene one after the other.

Though the semantic context of the animation is poor, observers of the four frames of figure 1 will often interpret the displacement of the two squares as a story of intentional action $[28,30]$ such as, for instance, "Before leaving, two boys (the squares) go tell their mum (the circle) goodbye." How can such an interpretation emerge from contextual categorization? C.A.D.S. models perceived action by building a contextual network of categories for each of the successive frames.

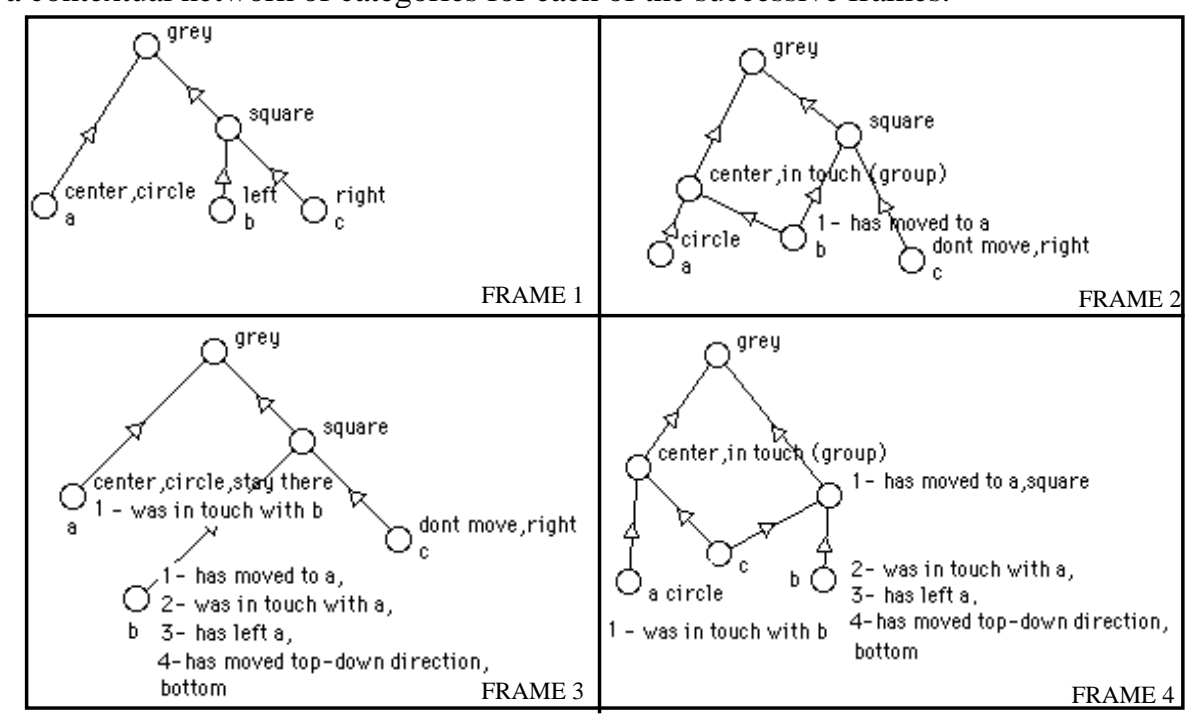

Figure 2. The Galois lattices of C.A.D.S.: successive networks of contextual categories provide support for action attribution (see text for a detailed explanation).

Frame 1: The network of frame 1 corresponds to a participant's description: there are three gray figures: $a, b$, and $c$ (the most superordinate category is "gray"), two squares: $a$ and $b$, (subordinate category: "square"), one on the left (subordinate category "left" of which object $b$ is an instance) and one on the right (subordinate category "right" of which object $c$ is an instance), and a circle in the center (category "center, circle" of which object $a$ is an instance).

Frame 2: Comparing lattices of frame 1 and of frame 2, the new perceptual data is that objects $a$ and $b$ are now "touching in the center" (a position). From object $b$ 's change in space and time it can be inferred that $b$ "has moved toward" object $a$. This is an inference derived from contextual categorization. "Moving toward" object $a$ is a new property attributed to $b$. In addition, C.A.D.S. noticed that object $c$ "did not move". It noticed this because objects belonging to the same category (squares) should have the 
same properties. This is a principle of contextual categorization that has two effects: the economy of simple lattices and the attribution of features not perceived. So, object $c$ is attributed the property "hasn't moved". At this point, perceptions and inferences $(b$ moved to $a, c$ did not move) may interface with knowledge in long term memory about action ( $b$ "moved toward" $a$ : $b$ "went to meet" $a, c$ "did not move" while $b$ "went toward" $a$ : $c$ "watched $b$ moving toward" $a$; ...). In addition, action attribution furnishes kinds of objects that can do the action (animated objects, persons, for instance male and female because of their size difference, ...).

Frame 3: What is new in frame 3 is that the group in the center no longer exists because object $b$ has moved to the bottom (its place has changed from center to bottom). So $b$ has left $a$ and $a$ is staying put. Now an observer can summarize and compare the changes in time and space having occurred from frames 1 to 3 , and say that "one of the two squares has gone to see the circle and left it".

Frame 4: Processing frame 4 with C.A.D.S. yields the interpretation that that $c$ has moved toward $a$ just as $b$ did earlier. Then C.A.D.S. will predict that $c$ will touch $a$, leave $a$, and move downwards, because of the generalizing procedure that helps infer unseen features. Successive interpretations such as "before leaving, two boys go say goodbye to their mum" can be inferred from "going toward somebody who doesn't change his/her place, touching, going away from him/her, etc". Notice that the property "gray" common to all three objects is filtered out by location in the most superordinate category, an observer might not even mention it.

Our point of view is that C.A.D.S.'s modeling of action perception may be a relevant representation of psychological process. The main idea is that perceived action is in the scope of on-line contextual categorization: networks of contextual categories are built from what is perceived at each moment; inferences concerning actions are produced from comparing categorical changes of objects in space and time. This approach is new and so is the idea that the variation in space and time of the properties of objects is what leads to the attribution of action to objects seen as agents [28].

\subsection{On line contextual temporal categorization in Galois lattices}

As seen in the preceding section, information already activated (t-1) and current properties ( $\mathrm{t}$ ) feed into a process that creates contextual categories by activating properties of the relationships of the objects to each other (in the previous example, the two squares). The hierarchical structure of the contextual categories allows the system to eliminate current state properties that are not considered relevant to the goals attributed ${ }^{2}$ (future).

Thus contextual properties appear in the network and the selection of relevant properties is mainly guided by the data furnished by the temporal context. In this way,

\footnotetext{
${ }^{2}$ The predictive ability that comes into play during understanding of actions performed by others, is based on the possibility of attributing goals to agents [e.g.; 14]. This allows excluding or considering action possibilities as well as facilitating comprehension of what is taking place. However, though most models agree on the fundamental role played by attributing goals to agents in order to recognize and determine the type of action that is being carried out, they do not explain how the prerequisite role of agent or patient is attributed to objects in the situation [30].
} 
the observer is in a position to infer a set of possible and imaginable actions, compatible with the properties already activated as the event has unfolded (t-1), with the properties of the current state of the situation $(\mathrm{t})$ and with the properties anticipated through the attribution of goals to agents $(t+1)$. For instance, the agent moving in the direction of a car could be seen as "going to use the car", but if this agent was previously seen "leaving the car", and is now seen "moving back to the car", it might be categorized as someone having forgotten something in the car. In thise case, the goal attributed will be "going to get something" instead of "going to drive the car".

Through temporal contextual categorization, the observer establishes coherence between different sequential events and adapts her perception of the current situation (bottom up process) according to the goals she has attributed to the agents (top-down process). In order to test the plausibility of our hypothesis about contextual categorization and the psychological relevance of the C.A.D.S. model, we are currently conducting a computer simulation using StarLogo software which takes the form of the computer model, ACACIA (Action by Contextually Automated Categorizing Interactive Agents)

\section{Simulating ACACIA's Contextual Categorization with StarLogo Software}

According to AL's basic principles, StarLogo 3 language makes it possible to populate a microworld with large numbers of autonomous agents. Agents (or "turtles", in StarLogo jargon) have properties, like their positions in a two-dimensional world, their headings, or any variable that the programmer wants to define for them, (e.g., their "closeness" to other agents). An "observer" controls how the system is set up, and is in charge of displaying the global properties of the agents. Thus, the "observer" may "spy on" the properties of each individual agent. Also, individual agents may have access to the properties of other individual agents, and act in consequence. For example, if agent A knows that agent B is now closer to A than before, then A could react by increasing its distance from $B$. The agents are then set loose to interact with each other, leading to complex emergent behavior.

We are presenting a simple situation to illustrate the programming rationale we are developing for simulating the emergence of interpretation in terms of action based on the temporal and spatial displacements of agents. We have defined three agents represented by three squares of three different colors. For each time unit, the agents move in space and construct a representation of perceived events in an output file. The knowledge in terms of action that each agent acquires is coded by a list of properties. These properties are relative to the agent's own position in space, the other agent's positions and their respective relational positions at each instant. Representations are updated for each agent and for each time unit according to certain probabilistic rules. Thus, at each instant, each agent has its own representation of the situation in accordance with what it "has learned" up to that point. This allows

\footnotetext{
${ }^{3}$ StarLogo is a software developed at the MIT Media Lab by Resnick, [18], specifically aimed to the simulation of decentralized, leaderless, and parallel multi-agent sytems, in which the interaction among the agents makes emerge global properties that are not specified in (and cannot derived analitically from) their local interaction rules.
} 
showing that changes in simple spatial coordinates can produce inferences in terms of perceived action such as "touching" and "approaching" through successive contextual categorization and applying probabilistic rules.

\subsection{Building the knowledge network}

In order to implement contextual categorization based on the Galois lattice in our StarLogo simulation (called ACACIA: Action by Contextually Automated Categorizing Interactive Agents), one linked data list is defined for each agent. At the outset, that is, before interpretation of action starts, the data lists are empty. As interpretation progresses, elements are added to the list or updated, resulting in a list with as many elements as properties of the agents are considered (see also footnote 1). Taking all the agents altogether, the lattice is represented as a matrix of $\mathrm{n}$ rows (one per agent) and $m$ columns (one per property). When the agent has a certain property (e.g., "being on the left"), then it is coded as "1" in the corresponding column, and for all the other agents not sharing that property (i.e., "not being on the left") it is coded "0". By using linked data lists it is possible to add, delete, insert, and update elements to the list, and so we can simulate how the Galois lattice grows when knowledge about other agents and their interaction with the environment is acquired by each agent.

At each time unit, the data list for an agent is compared with its own data list at the preceding time unit, in order to check for changes in the properties. For instance, if at t-1 the value for the properties "being on the left" and "being on the right" are " 1 " and " 0 " respectively, but they are " 0 " and " 1 " at $t$, then a new property is added (if not already present), i.e., "moved from left to right", and an "1" is assigned for the agent. All the other agents not sharing that new property at $t$ are assigned "0" (i.e., "not moved from left to right"). In this way, new interpretations are added to the knowledge network, which are superimposed on previous or more primitive ones. For a given set of possible locations (left, right, center), movements (from left to right, etc.), and relative movements among agents (approach, leave, in touch, etc.), the number of possible properties, and the rules used for generating upper level interpretations from lower level ones can be specified in advance.

The agents' interpretations are obtained in an "output" file listing the contents of the semantic network in the form of the properties that each agent has recorded. This simultaneously represents its knowledge and interpretation of the situation at each instant.

The process of contextual categorization operates at different levels of specificity. It operates in parallel for each agent's construction of the representation at each moment and it works sequentially and locally in activating emerging properties (including what an object can do) as a function of what an agent has learned from its context. For more sophisticated simulation of action interpretation using our ACACIA model, we are currently developing computer models 1) of the probability of perceived events and properties in the situation, and 2) to refine the simple rules defining basic displacements in space (i.e. walking at random, chasing each other, approaching and leaving all the other agents, as in the P-Space model) $[16,17]$. 


\subsection{Probability, noise and generalization}

When modeling the interpretation of actions, it is not always necessary to assume that the information gathered by the agents, and the rules they apply in order to produce interpretations, are error free. In fact, more realistic simulations can be achieved by allowing for some noise in the data. When an agent perceives other agents' actions, it might perceive them incorrectly, or it even might not perceive them at all. Thus, in some cases the perceptions can be incomplete or absent. This may be true for some of the interacting agents but not for others. For example, if we define a scope of perception for each agent by specifying some area in front of it to which it can pay attention, then, depending on its current heading, the actions of the agents lying outside its current scope will be missing from the agent's current knowledge network; that is, neither a " 1 " nor a " 0 " can be assigned to a property the agent is unaware of. Consequently, whereas some agents could reach interpretations based on "correct" perceptions, others may interpret using incomplete ones. The rules for using perceived actions for interpretation should thus be probabilistic.

Probability plays yet another role. In order to generalize (e.g., "all blue agents move from left to right, except if they are approached by a red agent, in which case they move from top to bottom"), an agent can either perceive the actions once, or perceive them many times. In the former case, a generalization that is based on one or several actions will have a low prediction power (that is, when applied in the future it will fail most of the time), while in the latter, powerful predictions will be possible. The agents can then make generalizations, test them, and assign them a degree of success in prediction. This way, the agents learn to categorize and generalize. The process of categorization and generalization is thus an emergent property of the agent's individual rules of movement and interpretation.

\subsection{Adaptation and Prediction}

Initially, the agents move according to a few simple pre-specified rules (i.e., agents may walk at random, chase each other, approach and leave all the other agents,...) $[16,17]$. However, as the simulation progresses and they learn how to predict other agents' actions through generalization, they become capable of reacting to or even anticipating these actions. Their movement is no longer governed by "blind rules" but by prediction and anticipation built on repeated series of interpretations of the other agents' actions. As a consequence, ACACIA uses the knowledge network specified in a Galois lattice for coordinated actions in a group of agents. Each agent acts as an observer and interpreter of all the other agents' actions, and it is the interaction of their interpretations and predictions that can be considered as an emerging property of group behavior.

\section{Conclusions}

As ACACIA integrates bottom-up (perceptual components) and top-down (cognitive components) processing to account for environmental and temporal context through successive constructions of contextual categories, the model can interpret the behavior 
of other agents without a knowledge base necessarily pre-defined by its designer. Its comprehension emerges from active situated cognition, organized in the form of semantic property networks in which actions are represented as properties inferred from modifications in the network's own "ad hoc" categories. As the situation evolves and creates spatiotemporal changes, the system is capable of changing its current representation of the situation as well as associating perceived events to the set of properties that constitute an object involved in the event through probabilistic calculations. In this way it is capable of interpreting but also of anticipating what is going to happen as a function of acquired knowledge about the action "possibilities" for other agents.

The system accounts for context (environmental and temporal) through: (i) comparing contextual category networks constructed at " $t$ " and " $t+1$ " to detect changes, (ii) a generalization process based on past categorization experience, a feature which endows it with predictive capacities. It is important to emphasize that the probabilistic rules which give ACACIA its predictive power are not contained in activated contextual schemas as in Intille and Bobick's $[9,10]$ model, rather they are highly dependant on knowledge recently acquired by the system in its context.

Acknowledgments: The research reported herein was supported by the CNRS (France) and a Comissionat per a Universitats i Recerca (Catalan Autonomous Government) Grant 1997SCR-00344. The authors are grateful to Elizabeth Hamilton for her reviewing of this paper, suggestions and critical comments.

\section{References}

[1] Ayes, D., \& Shah, M. (1998): Monitoring Human Behavior in an Office Environment. In Proceeding of the IEEE Computer Society Workshop: The Interpretation of Visual Motion, CVPR'98, Santa Barbara, CA, June 22. 65-72.

[2] Barbut, M., \& Monjardet, B. (1970). Ordre et Classification: algèbre et combinatoire. Paris. Hachette.

[3] Barsalou, L.W. (1983). Ad Hoc Categories. Memory and Cognition, 11, 211-227.

[4] Barsalou, L.W. (1991). Deriving categories to achieve goals. In G.H. Bower (Ed.), The psychology of learning and motivation: Advances in Research and Theory, Vol. 27, (164). New York: Academic Press.

[5] Brooks, R.A. (1991). Intelligence without representation. Artificial Intelligence, 47, 139-159.

[6] Cordier, F., \& Tijus, C. A. (2000). Propriétés d'Objets: Typologie et Organisation. Actes du XXVI/ Congrès International de Psychologie, Montréal, 26-29 juillet 2000.

[7] Gibson, J. (1977). The Theory of affordance . In R.E. Shaw, \& J. Brandsford (Eds.) Perceiving, acting, and knowing. Hillsdale, N.J.: Erlbaum

[8] Gibson, J. (1979). The Ecological Approach to Visual Perception. Boston, M.A.: Houghton Mifflin.

[9] Intille, S. S. (1999). Visual recognition of Multy-Agent action. Ph.D. Thesis, Massachusetts Institute of Technology.

[10] Intille, S. S., \& Bobick, A.F. (1998). Representation and Visual Recognition of Complex, Multi-agent Actions using Beliefs Networks. In Proceeding of the IEEE Computer Society Workshop: The Interpretation ofVisual Motion, CVPR'98, Santa Barbara, CA, June 22. 73-80. 
[11] Langton, C.G. (1989). Artificial life. In C.G. Langton (Ed.), Artificial life. The proceedings of an interdisciplinary workshop on the synthesis and simulation of living systems (pp.1-47). Redwood City, Ca: Addison-Wesley.

[12] Maes, P. (1998). Modeling adaptive autonomous agents. In C.G. Langton (Ed.), Artificial life. An overview (pp. 135-162). MIT Press.

[13] Miller, G. A., \& Johnson-Laird, P. N. (1976). Language and Perception. Cambridge, MA: Harvard Univ. Press.

[14] Oatley, K. \& Yuill, N. (1985) Perception of personal and interpersonal action in a carton film. British Journal of Social Psychology 24, 115, 124.

[15] Poitrenaud, S. (1995). The Procope Semantic Network: an alternative to action grammars. International Journal of Human-Computer Studies, 42, 31-69.

[16] Quera, V., Solanas, A., Salafranca, L., Beltran, F.S., \& Herrando, S. (2000). A dynamic model for inter-agent distances. In Meyer, J.-A., Berthoz, A., Floreano, D., Roitblat, H.L., Wilson, S. (Eds.), From Animals to Animats 6, SAB2000 Proceedings Supplement. Honolulu (Hawaii): USA.

[17] Quera, V., Solanas, A., Salafranca, Ll., Beltran, F.S., \& Herrando, S. (2000). PSPACE: A program for simulating spatial behavior in small groups. Behavior Research Methods, Instruments, and Computers, 32 (1), 191-196.

[18] Resnick, M. (1994). Turtles, Termites, and Traffic Jams: Explorations in Massively Parallel Microworlds. Cambridge, MA: MIT Press.

[19] Richard, J.F., \& Tijus, C. A. (1998). Modeling the Affordances of Objects in Problem Solving. In A.C. Quelhas \& F. Pereira (Ed.), Cognition and Context. Lisboa ISPA, 293315.

[20] Ross, B.H. (1996). Category learning as problem solving. In D.L. Medin (Ed.), The Psychology of Learning and Motivation: Advances in Research and Theory, Vol. 35 (165-192). San Diego, CA: Academic Press.

[21] Schyns, P., Goldstone, R.L., \& Thilbaut, J.-P. (1998). The development of features in object concepts. Behavioral and Brain Sciences 21 (1): 1-54.

[22] Thibadeau, R. (1986). Artificial Perception of Actions, Cognitive Science, 10, 177 149.

[23] Tijus, C. A., \& Moulin, F. (1997). L'assignation de signification étudiée à partir de textes d' histoires drôles. L'Année Psychologique, 97, 33-75.

[24] Tijus, C. A., \& Poitrenaud, S. (1997). Modeliser l'Affordance des Objets. Actes du 6ème colloque: Sciences Cognitives, Individus et Société, p 57-65.

[25] Tijus, C.A., (1996). Assignation de signification et construction de la réprésentation. Habilitation à diriger les recherches. Université de Paris 8.

[26] Turner, R. M. (1999). Model of Explicit Context Representation and Une for Intelligent Agents. In J.G. Carbonell \& J. Siekmann (eds), Lectures Notes in Artificial Intelligence, vol. 1688, Modeling and Using Context, (pp. 375-388). New-York: Springer.

[27] Yamauchi, T., \& Markman, A. (1998). Category Learning by Inference and Classification. Journal of Memory and Language, 39, 124-148.

[28] Zibetti, E., Hamilton, E. \& Tijus, C.A. (under revision). Contextual Categorization in Interpreting Perceived Actions: The Role of Objects Properties. Cognitive Science.

[29] Zibetti, E., Hamilton, E., \& Tijus C.A. (1999). The role of Context in Interpreting Perceived Events as Action. In J.G. Carbonell \& J. Siekmann (eds), Lectures Notes in Artificial Intelligence, vol. 1688, Modeling and Using Context, (pp. 431-441). NewYork: Springer.

[30] Zibetti, E., Poitrenaud, S., \& Tijus, C.A. (in press). La construction de la représentation de l'action perçue. Intellectica. 